\title{
Neutron Detection in Mixed Neutron-Gamma Fields with Common Nal(TI) Detectors
}

This paper was downloaded from TechRxiv (https://www.techrxiv.org).

LICENSE

CC BY-NC-SA 4.0

SUBMISSION DATE / POSTED DATE

$21-12-2021 / 23-12-2021$

\section{CITATION}

Pausch, Guntram; Kreuels, Achim; Scherwinski, Falko; Kong, Yong; Küster, Mathias; Lentering, Ralf; et al. (2021): Neutron Detection in Mixed Neutron-Gamma Fields with Common Nal(TI) Detectors. TechRxiv. Preprint. https://doi.org/10.36227/techrxiv.17317175.v1

$\mathrm{DOI}$

10.36227/techrxiv.17317175.v1 


\title{
Neutron Detection in Mixed Neutron-Gamma Fields with Common NaI(Tl) Detectors
}

\author{
Guntram Pausch, Member, IEEE, Achim Kreuels, Falko Scherwinski, Yong Kong, Member, IEEE, \\ Mathias Küster, Ralf Lentering, Member, IEEE, Andreas Wolf, and Juergen R. Stein, Member, IEEE
}

\begin{abstract}
NaI(TI) scintillators can detect thermal neutrons with surprising efficiency, even in the presence of ambient gamma radiation. This is due to a peculiarity of the ${ }^{128}$ I level scheme. De-excitation cascades following neutron captures in ${ }^{127} \mathrm{I}$, a main constituent of $\mathrm{NaI}$ with quite large neutron capture crosssection, unexpectedly often involve the $167.4 \mathrm{keV}$ state of ${ }^{128} \mathrm{I}$ with $175 \mathrm{~ns}$ half-life, which feeds another isomeric level at $137.8 \mathrm{keV}$ having a half-life of $845 \mathrm{~ns}$. The $29.5 \mathrm{keV}$ transition between both levels, as well as the subsequent de-excitation sequence to ground state comprising $137.8 \mathrm{keV}$ sum energy, are almost certainly absorbed in the crystal. Signals including three scintillation pulses within few microseconds, the first one caused by a prompt part of the cascade, the second and third one disclosing $30 \mathrm{keV}$ and $138 \mathrm{keV}$ energy depositions and characteristic delays, are clearly related with such neutron captures. Double-pulse sequences comprising only the primary pulse and a delayed $138 \mathrm{keV}$ signal are also distinctive but more prone to loaddependent background by random pulse pileups. The paper demonstrates neutron detection with a $\varnothing 2 " \times 2 " \mathrm{NaI}(\mathrm{Tl})$ scintillation detector by finding and analyzing such double- and triple-pulse sequences in digitized detector signals. It quantifies effect and background rates as a function of the detector load ranging up to $30 \mathrm{kcps}$. Complementary measurements with a ${ }^{6} \mathrm{Li}$ co-doped Ø2" $\times 2 " \mathrm{NaI}(\mathrm{TI})\left(\mathrm{NAIL}{ }^{\mathrm{TM}}\right)$ scintillator allow relating the neutron detection yield by delayed-coincidence counting with the ${ }^{6} \mathrm{Li}$ capture rate in the same crystal, and thus to evaluate prospects and limits of this technique.
\end{abstract}

Index Terms - Scintillator, neutron detector, gamma detector, gamma spectroscopy, digital signal processing.

\section{Motivation}

$\mathrm{N}$ EUTRON detection is a standard task in many fields of research, industry, and administration. Among other areas, neutron detectors are needed for basic research as nuclear or solid-state physics, for monitoring of radiation fields at nuclear reactors, accelerators, medical facilities or in the environment, and for searching suspect radiation sources in nuclear safeguards or homeland security applications. Here,

Manuscript, December 21, 2021.

Guntram Pausch (e-mail: guntram.pausch@target-sg.com), Achim Kreuels (e-mail: achim.kreuels@target-sg.com), Falko Scherwinski (e-mail: falko.scherwinski@target-sg.com), Yong Kong (e-mail: yong.kong@targetsg.com), Mathias Küster (e-mail: mathias.kuester@target-sg.com), Ralf Lentering (e-mail: ralf.lentering(a)target-sg.com), Andreas Wolf (e-mail: andreas.wolf@target-sg.com), and Juergen R. Stein (e-mail: juergen.stein(target-sg.com) are with Target Systemelektronik GmbH \& Co. KG, Heinz-Fangman-Straße 4, 42287 Wuppertal, Germany. an enhanced rate of neutron detections indicates Special Nuclear Materials (SNM) comprising fissile nuclides and traces of isotopes undergoing spontaneous fission. This signature is of great value for an unambiguous identification of SNM and a desirable supplement to the gamma-ray spectrum that finally discloses the nuclide vector. Handheld or backpack radiation detection systems for homeland security applications are therefore expected to not only provide gamma-ray spectroscopy for radionuclide identification, but at best also means for detecting (few) neutrons in the presence of (strong) ambient gamma radiation [1] [2].

Until the emerging shortage of worldwide ${ }^{3} \mathrm{He}$ reserves and a subsequent ${ }^{3} \mathrm{He}$ price explosion around 2008, neutron detection in nuclear safeguards and security had been based essentially on ${ }^{3} \mathrm{He}$ tubes embedded in moderating materials [3]. The "Helium-3 crisis", however, combined with corresponding funding programs, triggered the development of alternative technologies [4]. $\mathrm{BF}_{3}$-filled or boron-lined proportional counters and ${ }^{6} \mathrm{LiF}-\mathrm{ZnS}$-coated plastic fibers or panels became reasonable alternatives for the large ${ }^{3} \mathrm{He}$ tubes used so far in radiation portal monitors [5]. Another line of research pushed the development of scintillator materials providing pulse-shape discrimination (PSD) capabilities and reasonable energy resolution for gamma-ray spectroscopy but comprising lithium or boron, preferably enriched or separated ${ }^{6} \mathrm{Li}$ or ${ }^{10} \mathrm{~B}$, as constituent or dopant. These so-called "neutrongamma scintillators" can detect thermal neutrons via neutron capture reactions in ${ }^{6} \mathrm{Li}$ or ${ }^{10} \mathrm{~B}$ generating pairs of energetic heavy ions, namely tritons and alpha particles or alpha particles and ${ }^{7} \mathrm{Li}$ recoils with sum energies of $4.78 \mathrm{MeV}$ or 2.31 to $2.79 \mathrm{MeV}$, respectively [6], which are stopped in the crystal. The corresponding scintillations are distinguished from gamma ray (i.e., electron) signals by means of pulseshape discrimination. The large thermal-neutron capture crosssections of ${ }^{6} \mathrm{Li}(940 \mathrm{~b})$ or ${ }^{10} \mathrm{~B}(3,840 \mathrm{~b})$ [6] warrant high neutron absorption probabilities and thus high neutron detection efficiencies even at low concentrations, e.g., around 1 at $\%$, of the corresponding isotope in the scintillation crystal. Prominent examples of such materials are $\mathrm{Cs}_{2} \mathrm{LiYCl}_{6}(\mathrm{Ce})$ (CLYC) [7] [8], $\mathrm{Cs}_{2} \mathrm{LiLaBr}_{6}(\mathrm{Ce})$ (CLLB) [9], and ${ }^{6} \mathrm{Li}$-codoped $\mathrm{NaI}(\mathrm{Tl})\left(\mathrm{NaIL}^{\mathrm{TM}}\right)$ [10].

Neutron-gamma scintillators seem just tailored for an application in Handheld Radionuclide Identification Devices (RIDs) or Backpack Radiation Detection Systems (BRDs). They allow combining gamma-ray spectroscopy and efficient 
neutron counting in a single detector, which reduces complexity, size, and weight of an instrument if compared with systems comprising a separate neutron sensor. They are, however, also much more expensive than sodium iodide scintillators of comparable size. Finally the market of RIDs and BRDs is still dominated by instruments based on common $\mathrm{NaI}(\mathrm{Tl})$. It is therefore useful to ask whether or not $\mathrm{NaI}(\mathrm{Tl})$ detectors could also provide at least some neutron sensitivity.

This is by far not a new idea. It has been well known that ${ }^{127} \mathrm{I}$, the only stable isotope of iodine and a main constituent of sodium iodide, is distinguished by a quite large thermalneutron capture cross-section of $6.2 \mathrm{~b}$ [11]. This means the neutron capture rate in ${ }^{127} \mathrm{I}$ of a common $\mathrm{NaI}$ crystal is roughly comparable with the capture rate in the ${ }^{6} \mathrm{Li}$ replacing about $1 \%$ of the sodium in a NaIL ${ }^{\mathrm{TM}}$ crystal of the same size. However, a neutron capture in ${ }^{127} \mathrm{I}$ generates a cascade of gamma rays (and, maybe, conversion electrons) with a sum energy corresponding to the neutron separation energy of ${ }^{128} \mathrm{I}$, about $6.83 \mathrm{MeV}$ [12], but no charged particle. This excludes pulse-shape discrimination as a means for separating neutron from gamma-ray detections and raises the question how to distinguish between the neutron-capture and the ambient gamma rays.

So far, this challenge has been tackled in two ways. A first method, named Neutron Capture Detector (NCD) and proposed more than ten years ago [13] [14], rests upon the fact that common radioactive sources quite rarely emit gamma quanta of an energy above $2,614 \mathrm{keV}$. Detector signals indicating an energy deposition between $\approx 3 \mathrm{MeV}$ and an upper limit close to the neutron separation energy $S_{n}\left({ }^{128} \mathrm{I}\right)$ can therefore be attributed to neutron captures in ${ }^{127} \mathrm{I}$. Disturbing background, which is essentially due to cosmic radiation, must be monitored and considered (for instance by extending the range of spectrometry far above $S_{n}$ and using the high-energy range for background normalization) or eliminated by other means [13] [15].

A second method, discussed by Yakushev et al. in 2017 [16], exploits delayed coincidences of consecutive signals in a single $\mathrm{NaI}(\mathrm{Tl})$ crystal. As known from previous work by Sakharov et al. [17], gamma-ray cascades following neutron capture in ${ }^{127} \mathrm{I}$ sometimes involve the $137.8 \mathrm{keV}$ state in ${ }^{128} \mathrm{I}$, which has a long half-life of 845 ns and decays to ground state via sequential emission of low-energy gamma rays or conversion electrons [18]. Those are almost certainly absorbed within the $\mathrm{NaI}$ crystal and generate a signal of about $138 \mathrm{keV}$ sum energy, following the prompt part of the cascade with a characteristic delay. Yakushev et al. searched time-stamped list-mode data, acquired with a $\varnothing 2.5 " \times 2.5 " \mathrm{NaI}(\mathrm{Tl})$ detector exposed to ambient radiation in a laboratory, for couples of consecutive pulses with delays in the microsecond range. They found the expected $845 \mathrm{~ns}$ decay component with a delayed signal in the energy range around $138 \mathrm{keV}$ and related this component to the ambient thermal neutron flux. The method failed, however, in gamma radiation fields causing random delayed coincidences between gamma-ray signals that finally cover the neutron signature. One obvious reason was the independent handling of prompt and delayed signals combined with the $1.8 \mu$ s trigger hold-off [16], which reduced the rate of detectible delayed signals with respect to the original rate at the given 845 ns half-life by a factor of 4-5.

Recently, Target Systemelektronik initiated research to enhance Yakushev's technique and to finally implement it in RIDs as Target's F500' (equivalent with Teledyne FLIR's identiFINDER ${ }^{\circledR} \quad \mathrm{R} 440^{2}$ ) or BRDs as Target's F900 ${ }^{3}$ (equivalent with Teledyne FLIR's identiFINDER ${ }^{\circledR}$ R7004). The initial goal was a distinct reduction of the minimum time distance (delay) between pulses that still can be resolved and processed individually. The chosen approach of decomposing piled-up pulse sequences into their constituents, instead of considering only well-separated signals, should increase the fraction of detectible delayed (double) coincidences and thus the neutron detection yield. The analysis of experimental data, however, triggered the discovery of another, complementary approach promising a better rejection of random coincidences at higher detector loads. We observed that data taken at a neutron source comprised a substantial fraction of triple-pulse pileups with second and a third pulse components corresponding to energy depositions around $30 \mathrm{keV}$ and $138 \mathrm{keV}$, respectively. These characteristic sequences disappeared in the presence of only gamma radiation. An obvious interpretation was a decay involving two long-lived states. As a matter of fact, gamma-ray cascades following neutron capture in ${ }^{127} \mathrm{I}$ sometimes involve not only the $137.8 \mathrm{keV}$ ( $845 \mathrm{~ns}$ ) state of ${ }^{128} \mathrm{I}$ but also a second isomeric level of $175 \mathrm{~ns}$ half-life at $167.4 \mathrm{keV}$, which feeds the $137.8 \mathrm{keV}$ state with a strongly converted $29.5 \mathrm{keV}$ transition [17] [18]. Corresponding detector signals often comprise an initial pulse reflecting the prompt part of the cascade in addition to the signal components generated by the delayed decays. A triple-pulse sequence with energy depositions around $30 \mathrm{keV}$ and $138 \mathrm{keV}$ for the second and the third component, respectively, and time delays in accordance with the half-lives of the isomeric states, is a clear and distinctive signature of a neutron capture in ${ }^{127} \mathrm{I}$. Because of the finite light decay time of $\mathrm{NaI}(\mathrm{Tl})$ scintillators, around $230 \mathrm{~ns}$ for the principal light component at room temperature [19], the first and the second signal components are mostly, the second and the third components are often piled up. Pileup analysis and decomposition (instead of pileup rejection) is obviously the key for finding the delayed triple-coincidence events related with neutron captures in the digitized detector signals.

\footnotetext{
${ }^{1}$ https://target-sg.com/f500.html

2 https://www.flir.com/products/identifinder-r440/

${ }^{3}$ https://target-sg.com/f900.html

$4 \underline{\text { https://www.flir.com/products/identifinder-r } 700 /}$
} 
Once an analysis providing separate time stamps and energy depositions for piled-up signal components is implemented, the delayed triple-coincidence technique can be used in parallel with a delayed double-coincidence analysis enhancing Yakushev's idea by the inclusion of piled-up pulse sequences. Both methods are valuable complements to the NCD approach mentioned above. As long as a $\mathrm{NaI}(\mathrm{Tl})$ spectrometer deploys fast, direct detector current digitization with suitable resolution, and digital signal processing, all these techniques can be realized with dedicated signal and spectrum processing algorithms. They can be implemented by soft- and firmware extensions, without increasing the hardware expense. An evaluation of the neutron detection performance achievable in that way, including a comparison with state-of-the-art neutrongamma scintillators, is obviously interesting and useful.

\section{OBJECTIVES OF THIS WORK}

The paper demonstrates that neutron detection with common $\mathrm{NaI}(\mathrm{Tl})$ detectors based on Yakushev's delayed coincidence method [16] can be effectively improved by decomposing piled-up signals into their constituents instead of considering only non-overlapping pulse pairs. It also introduces and substantiates a complementary method based on delayed triple coincidences, distinguished by higher selectivity and better robustness against external gamma load. Neutron detection yields and effect-to-background ratios achievable with these techniques are quantified for a $\varnothing 2$ " $\times 2$ " $\mathrm{NaI}(\mathrm{Tl})$ operated in mixed neutron and gamma radiation fields up to detector loads of $30 \mathrm{kcps}$, and compared with the performance of a $\varnothing 2 " \times 2 "{ }^{\prime 6} \mathrm{Li}$-codoped $\mathrm{NaI}(\mathrm{Tl})$ detector.

\section{EQUiPMENT AND MethodS}

\section{A. Instrumentation, setup, and data acquisition}

A first set of measurements was performed with a Target F500 comprising a $\varnothing 2 " \times 2 " \mathrm{NaI}(\mathrm{Tl})$ scintillator read out with a Hamamatsu R10601 photomultiplier tube (PMT). A second set of measurements was accomplished with a Teledyne FLIR identiFINDER ${ }^{\circledR}$ R440 equipped with a $\varnothing 2 " \times 2 "{ }^{6} \mathrm{Li}$ co-doped $\mathrm{NaI}(\mathrm{Tl})$ crystal (NAIL ${ }^{\mathrm{TM}}$ ) by Saint Gobain Crystals ${ }^{5}$, also coupled with a Hamamatsu R10601. Both instruments are commercial handheld Radionuclide Identification Devices (RIDs) with identical electronics sampling the PMT anode current directly with 14 bit resolution at a rate of $250 \mathrm{MS} / \mathrm{s}$. They were operated with a modified firmware by Target Systemelektronik that allowed raw-data transfer of sampled signals via USB to a PC.

For a measurement the corresponding RID was fixed near a ${ }^{252} \mathrm{Cf}$ neutron source sitting in a polyethylene enclosure (Figure 1). Additional gamma sources were placed close to the RID to increase the detector load. Before starting raw-data acquisition, the actual count rate was determined and adjusted to the planned level by varying the distance of the extra

\footnotetext{
${ }^{5}$ https://www.crystals.saint-gobain.com/products/nai-sodium-iodide-tllithium
}

gamma sources to the detector. Once the detector load was in the target range, the collection of raw-data signals was started.

The data acquisition was controlled by a MATLAB script running on a PC connected to the RID via USB interface. Raw data were stored on a file server in consecutive event records,

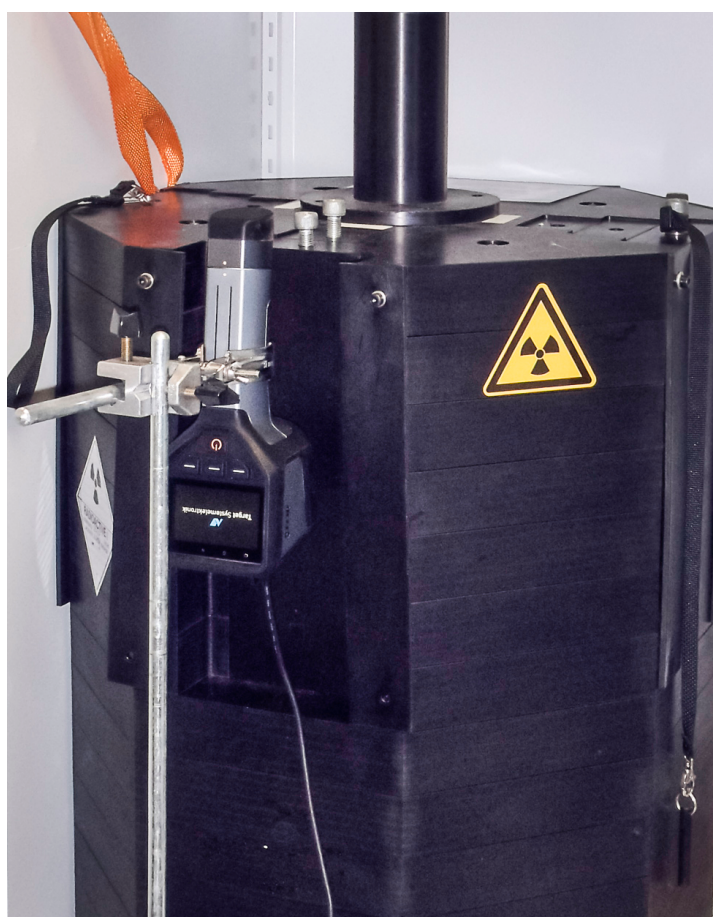

Figure1. Teledyne FLIR R440 fixed at the ${ }^{252} \mathrm{Cf}$ neutron source used for measurements at Target Systemelektronik in Wuppertal, Germany.

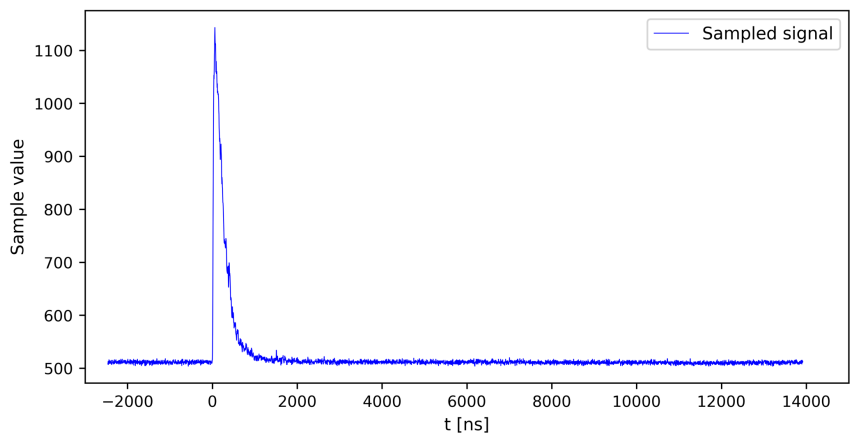

Figure 2. Exemplary raw data record of a single $\mathrm{NaI}(\mathrm{Tl})$ detector signal.

each comprising $4 \mathrm{k}$ of 14 bit samples corresponding to a $16 \mu \mathrm{s}$ time range. A record starts about 615 samples $(2.46 \mu \mathrm{s})$ ahead of the event trigger. An exemplary raw-data event is shown in Figure 2.

\section{B. Signal Processing and Detection of Delayed Coincidences}

All pulse processing and data analysis was performed offline by means of Python scripts.

First, the baseline of each raw-data event was shifted to zero by using the pre-trigger part of the signal as baseline reference. The leading and the main parts of the signal were then integrated to measure the corresponding pulse charges $A_{\text {lead }}$ and $A_{0}$. The integration range comprised $160 \mathrm{~ns}$ for the lead charge and $3.6 \mu \mathrm{s}$ for the main charge, starting $24 \mathrm{~ns}$ 
ahead of the actual trigger point, respectively.

The signal was then searched for delayed pulse components by successive stripping. A reference pulse, determined from the same measurement by summing up baseline-corrected signals passing rough amplitude and pileup rejection filters, was normalized to yield the same lead charge as the actual signal (Figure 3). The normalized signal was stripped of the first pulse, and the resulting residuum was searched for a possible next trigger, starting at the sample following the actual lead integration range. The algorithm is impervious to a trigger within the lead integration range. The minimum time distance $\Delta t_{\min }$ between sub-pulses that can be detected and resolved by the stripping procedure is therefore about $160 \mathrm{~ns}$.

If yet another trigger was found, the main charge of the actual signal was corrected for the delayed contribution(s) obtained by integrating the residuum till the end of the actual main integration range. This yields more precise main pulse charges and (after calibration) energy values than an integration of the normalized mean pulse, as the normalization considers only the photoelectron statistics comprised in the lead charge which means a larger statistical uncertainty. Finally, the residuum was taken as the actual signal, and the stripping was repeated until no further trigger was found.

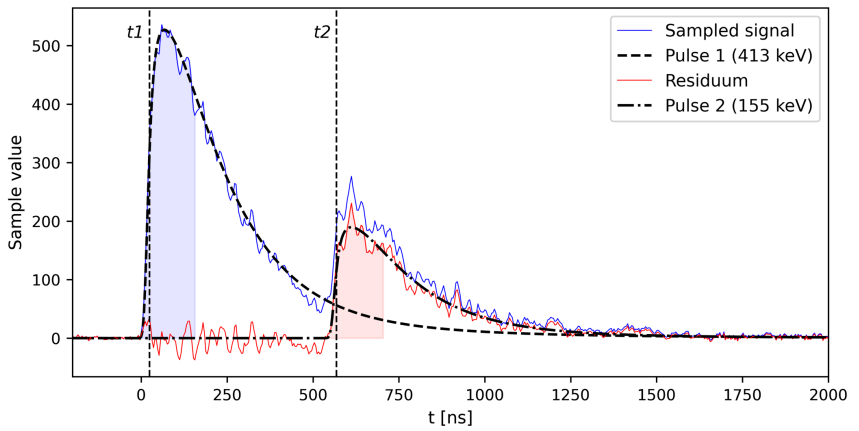

Figure 3. Illustration of the stripping procedure for an exemplary event: A mean pulse is normalized to the leading part of and then subtracted from the signal. The normalization range is shaded. The residuum is searched for a next trigger. Once a trigger is found, the stripping procedure is repeated for the residuum. Pulse 1 and Pulse 2 denote the signal components (normalized mean pulses) found in the exemplary event.

The result is a list of $M$ trigger points $t_{i}$ and sub-pulse charges $A_{i}$ per event, where $i$ denotes the sub-pulse index in and $M$ the multiplicity of the corresponding event. The subpulse charges $A_{i}$ were translated to energy depositions $E_{i}$ assuming a linear scaling between pulse charge and energy. The calibration was obtained from a reference measurement with a ${ }^{137} \mathrm{Cs}$ source, where the $662 \mathrm{keV}$ photopeak position was determined in a histogram of the primary pulse charge $A_{1}$. Note that $A_{1}$ equals $A_{0}$ if no further trigger (i.e., sub-pulse) is found in the main integration range of the primary signal.

All events were then searched for delayed sub-pulses $(i \geq 2)$ corresponding to energy depositions between 15 and $60 \mathrm{keV}$ (30 keV sub-pulses) or between 110 and $180 \mathrm{keV}$ (138 keV sub-pulses). Events comprising a $30 \mathrm{keV}$ sub-pulse followed by a $138 \mathrm{keV}$ sub-pulse or comprising a $138 \mathrm{keV}$ sub-pulse but no preceding $30 \mathrm{keV}$ sub-pulse were considered as delayed triple-coincidence (D3C) or delayed double- coincidence (D2C) events, respectively.

Exemplary pulse decompositions disclosing D3C events are shown in Figure 4. The middle picture points to a challenge: $30 \mathrm{keV}$ energy deposited in a $\mathrm{NaI}(\mathrm{Tl})$ scintillator generate just 300-360 photoelectrons in an attached PMT with standard bialkali photocathode yielding 10-12 photoelectrons per $\mathrm{keV}$ [20], statistically distributed in time [21]. The small signal is hard to find if it piles up with an (often much larger) primary signal. A low trigger threshold increases the sensitivity to D3C events but also the probability of false sub-pulse detections by random threshold crossings. Those may happen in the tail of every signal because of statistical fluctuations reflecting the random process of photoelectron generation over time.
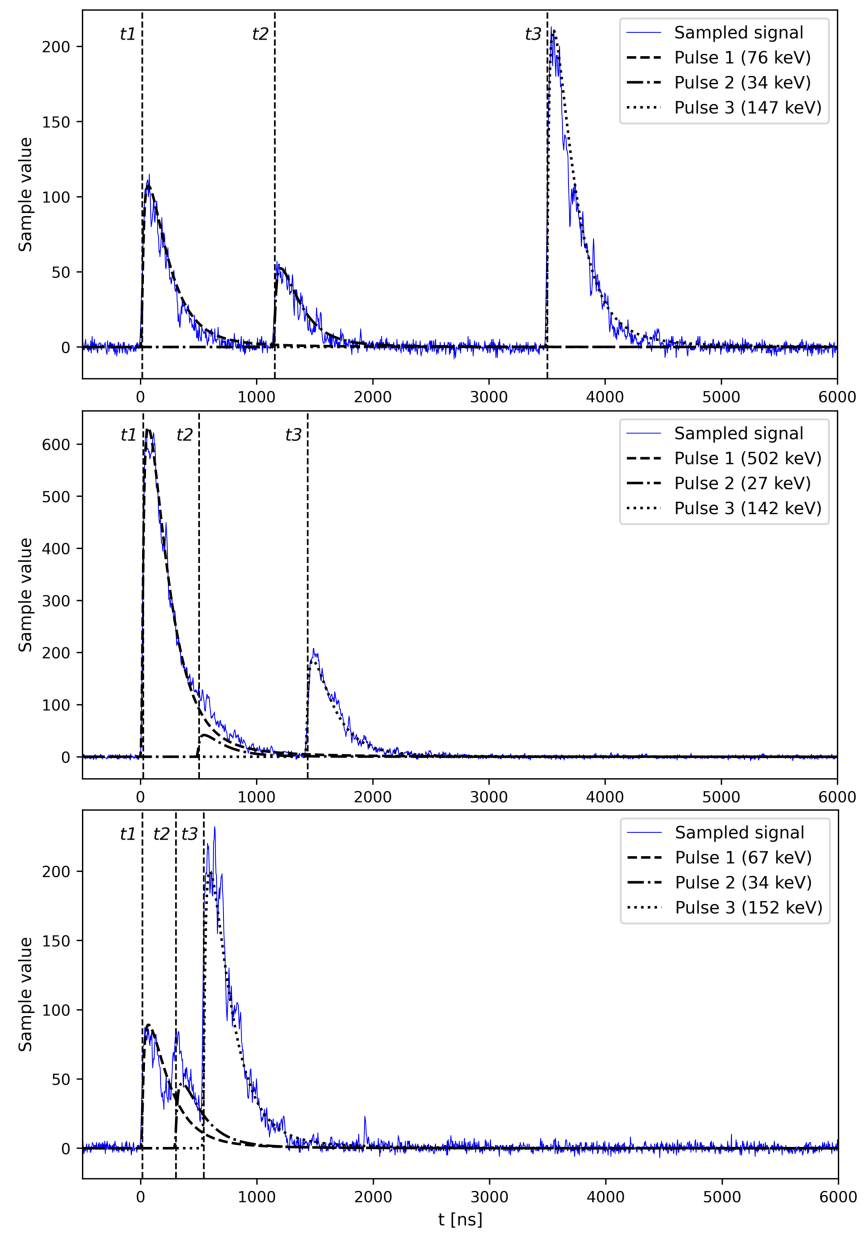

Figure 4. Exemplary pileup decomposition results yielding delayed triplecoincidence (D3C) sequences with delayed $30 \mathrm{keV}$ and $138 \mathrm{keV}$ sub-pulses. The upper panel shows a rare case of well separated components. Mostly, the second sub-pulse piles up with the first one and is hard to detect because of the low photoelectron number produced with an energy deposition of only $30 \mathrm{keV}$ (middle panel). Sometimes all three sub-pulses overlap (lower panel).

\section{Identification of ${ }^{6}$ Li Neutron Captures in NaIL ${ }^{\mathrm{TM}}$}

Data measured with the NaIL ${ }^{\mathrm{TM}}$ detector were also searched for neutron captures in ${ }^{6} \mathrm{Li}$. In addition to the signal processing described above, a pulse shape parameter PSD denoting the ratio of lead to main pulse charge of the primary signal component was computed for every event. Figure 5 shows a plot of this parameter versus the energy deposition $E_{1}$ corresponding to the primary signal component. Signals due to 
neutron captures in ${ }^{6} \mathrm{Li}$ were separated from gamma-ray signals by a rectangular cut as indicated in the figure.

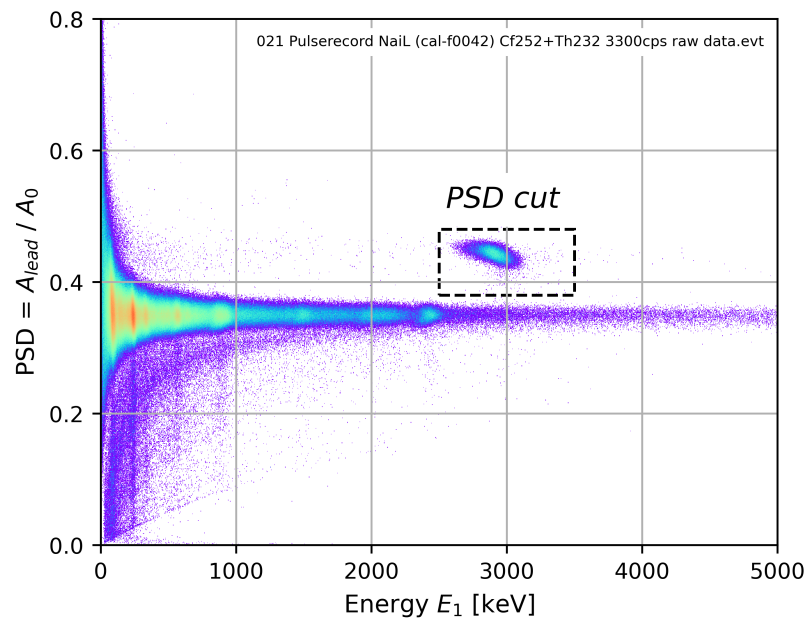

Figure 5. Plot of a pulse shape parameter $P S D$ versus the energy deposition $E_{1}$ corresponding to the primary signal component for a measurement with the NaiL ${ }^{\mathrm{TM}}$ detector exposed to moderated neutrons of the ${ }^{252} \mathrm{Cf}$ source and additional gamma rays from a ${ }^{232} \mathrm{Th}$ source generating an overall detector load of $3.3 \mathrm{kcps}$. A rectangular cut selects the ${ }^{6} \mathrm{Li}$ neutron-capture events.

\section{Monitoring of High-Energetic Neutron-Capture Signals}

For a comparison of different neutron detection modes, the high-energetic gamma-ray events indicating neutron captures according to the NCD principle were also monitored. Figure 6 exhibits energy spectra measured with the $\mathrm{NaI}(\mathrm{Tl})$ detector in the presence of gamma rays and neutrons from a ${ }^{252} \mathrm{Cf}$ source, or only gamma rays from a ${ }^{232} \mathrm{Th}$ source, at equal detector loads of $1.1 \mathrm{kcps}$. NCD "neutron" events were selected by a cut in the energy deposition $E_{1}$ of the primary signal component. The chosen limits, 3.5 to $8 \mathrm{MeV}$, maintain a safe

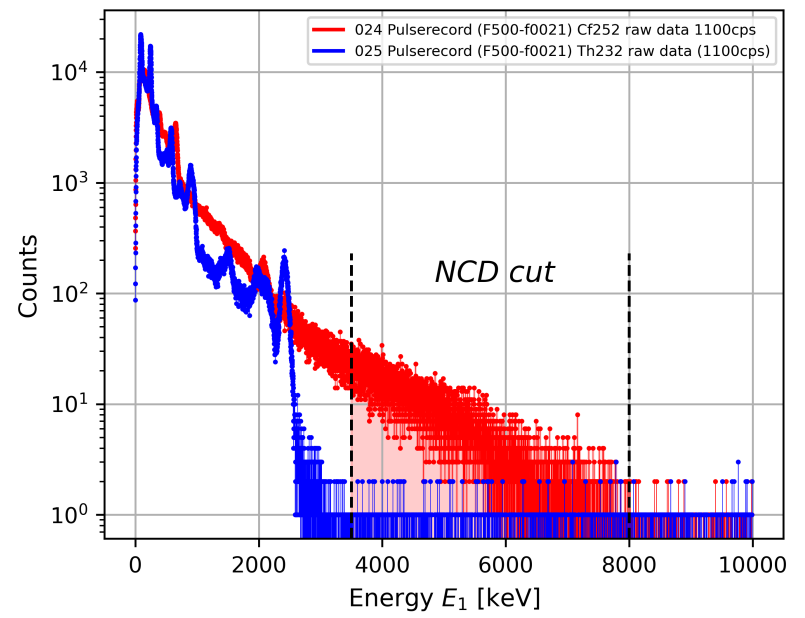

Figure 6. Energy spectra measured with the $\mathrm{NaI}(\mathrm{Tl})$ detector exposed to a ${ }^{252} \mathrm{Cf}$ source (in red) or to gamma rays from a ${ }^{232} \mathrm{Th}$ source (in blue). NCD events were selected by an energy cut. The shaded area indicates the highenergy events attributed to neutron-capture gamma-ray cascades. distance from the $2,614 \mathrm{keV}$ line of ${ }^{208} \mathrm{Tl}$ comprised in the ${ }^{232} \mathrm{Th}$ decay chain, and the maximum energy deposition of gamma rays following neutron capture in ${ }^{127} \mathrm{I}$, which equals the neutron separation energy of ${ }^{128} \mathrm{I}$.

The contribution of neutron-related events in the NCD window exceeds the background measured without neutron source by far, which confirms the usability of these counts as neutron indicator.

\section{E. Simulation of delayed coincidence signals}

Sets of signals comprising a prompt component and one or two of the delayed transitions in ${ }^{128} \mathrm{I}$ were simulated to estimate the efficiency of D2C and D3C detection with the given signal processing algorithm.

A simulated delayed triple-coincidence event consists of a prompt component, a second signal of $30 \mathrm{keV}$ with a random delay reflecting $175 \mathrm{~ns}$ half-life, and a third signal of $138 \mathrm{keV}$ delayed in accordance with a $845 \mathrm{~ns}$ half-life. A simulated delayed double-coincidence event consists of the primary component and a second signal of $138 \mathrm{keV}$ with a random delay according to $845 \mathrm{~ns}$ half-life. The energy deposition assumed for the prompt component is randomly chosen from a uniform distribution between 10 and $3,500 \mathrm{keV}$.

The simulation of the detector responses to a distinct, immediate energy deposition in the crystal followed the model described in reference [21]. It comprised

(i) The statistical generation of photoelectrons with a given yield per $\mathrm{keV}$ (phe/keV),

(ii) The random temporal distribution of the individual photoelectrons according to a rise time of $14 \mathrm{~ns}$ and decay components of $0.23 \mu \mathrm{s}$ and $1.0 \mu \mathrm{s}$ contributing $97 \%$ and $3 \%$ to the overall light emission of the scintillator, respectively,

(iii) The Poisson branching process of the photoelectrons at the first dynodes [22],

(iv) The convolution of the dynode electron bunches, considering the PMT gain and the distribution of the transit time spread.

The overall digitized signal was modeled as the superposition of responses to the distinct signal components, complemented with reasonable delta- and step-noise contributions in accordance with the noise level found in measured signals, convolved with the analog pre-sampling filter response of the RID electronics, and downscaled to the sampling resolution of the ADC used. Integral or differential nonlinearities of the ADC have not been considered.

The simulations were performed with a MATLAB script generating pulse records in raw-data format. These records were analyzed with the same Python scripts as used for the analysis of experimental data. 
Examples of simulated triple-pulse sequences are shown in Figure 7. The graphs once more illustrate the challenge of finding a $30 \mathrm{keV}$ sub-pulse in the statistically blurred tail of a larger primary signal.
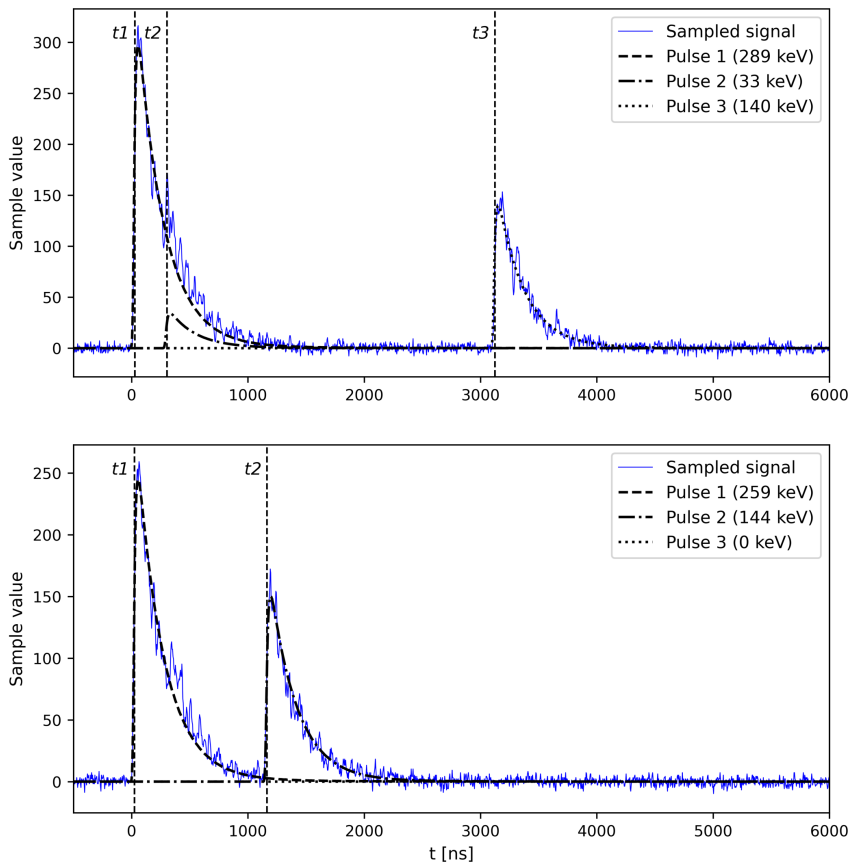

Figure 7. Exemplary delayed triple-pulse sequences simulated for a photoelectron yield of $10 \mathrm{phe} / \mathrm{MeV}$. In the first case (upper panel), the $30 \mathrm{keV}$ component was recognized by the signal processing algorithm. In the second case (lower panel), the algorithm missed it and the signal was interpreted as delayed double coincidence (D2C).

\section{RESUlts}

\section{A. Delayed Double and Triple Coincidences}

Figure 8 presents a scatter plot of the energy deposition $E_{2}$ comprised in the second signal component versus the time difference (delay) $t_{21}=t_{2}-t_{1}$ between the second and the primary signal components. The data were taken with the $\varnothing 2 " \times 2 " \mathrm{NaI}(\mathrm{Tl})$ detector exposed to the ${ }^{252} \mathrm{Cf}$ neutron source.

The plot shows a crest at $E_{2} \approx 150 \mathrm{keV}$ with fading intensity towards rising $t_{21}$ in accordance with a half-life around $1 \mu \mathrm{s}$. Delayed coincidence events as observed by Yakushev et al. [16] should appear as a ridge around $E_{2}=138 \mathrm{keV}$. The shift towards larger energies is caused by the scintillator's nonproportional light yield. Depending on doping level and integration time, $\mathrm{NaI}(\mathrm{Tl})$ crystals exhibit a light excess of $5-10 \%$ in the $20-150 \mathrm{keV}$ range [23]. Hence, a light pulse in the wake of $138 \mathrm{keV}$ energy deposition could appear as $\approx 150 \mathrm{keV}$ signal if a linear energy calibration with $662 \mathrm{keV}$ reference is used.

The graph exhibits a second, less pronounced, and shorter ridge around $30 \mathrm{keV}$. It turned out to comprise essentially triple-pulse events with the third component reflecting energy depositions around $138 \mathrm{keV}$. Both structures disappear in the absence of neutrons (Figure 9). The observations suggested that corresponding events represent detector responses to the prompt part, to a first delayed $(\sim 30 \mathrm{keV})$ transition, and to a second delayed transition, namely the known de-excitation of the $137.8 \mathrm{keV}$ level to ground state, which are part of the same gamma-ray cascade following neutron capture in ${ }^{127}$ I. Indeed, older [17] as well as more recent data published for the of ${ }^{128} \mathrm{I}$

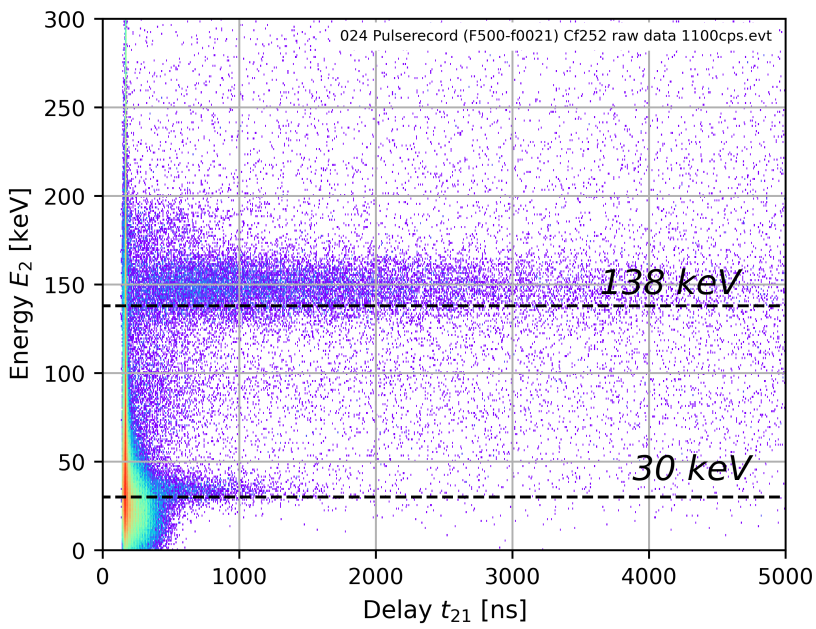

Figure 8. Energy deposition $E_{2}$ assigned to the second signal component versus the delay $t_{21}$ between the primary and the second signal components, plotted for all events of multiplicity $M \geq 2$ in Run 024 . The data were measured with the $\varnothing 2 " \times 2 " \mathrm{NaI}(\mathrm{Tl})$ scintillator, exposed to the ${ }^{252} \mathrm{Cf}$ neutron source, at a detector load of $1.1 \mathrm{kcps}$.

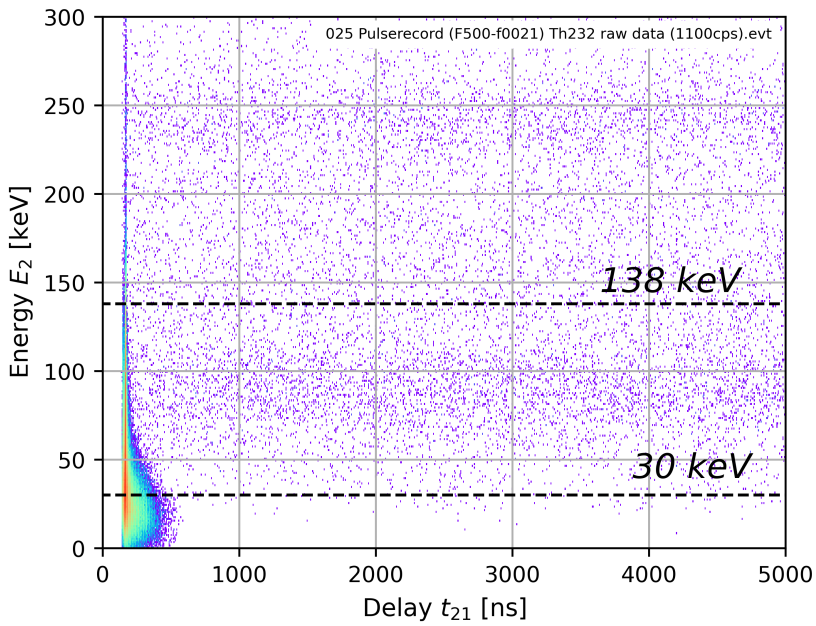

Figure 9. Energy deposition $E_{2}$ assigned to the second signal component versus delay $t_{21}$ between the primary and the second signal components, plotted for all events of multiplicity $M \geq 2$ in Run_025. The data were measured with the $\varnothing 2 " \times 2 " \mathrm{NaI}(\mathrm{Tl})$ scintillator, exposed to a ${ }^{232} \mathrm{Th}$ source, at a detector load of $1.1 \mathrm{kcps}$. Note the absence of the $138 \mathrm{keV}$ and $30 \mathrm{keV}$ ridges.

level scheme [18] match with this interpretation: There is a $167.4 \mathrm{keV}$ state of $175 \mathrm{~ns}$ half-life which decays to the $137.8 \mathrm{keV}$ state via an (almost) completely converted $29.5 \mathrm{keV}$ transition. The corresponding energy is surely absorbed within the NaI crystal. The $137.8 \mathrm{keV}$ level is, however, fed from other, promptly decaying states as well. Delayed double-coincidence events could therefore originate from cascades feeding this level promptly after neutron capture, or from cascades involving both isomeric states but generating detector signals with an unresolvable $30 \mathrm{keV}$ component. 
The $2 \mathrm{~d}$ distribution of the energy depositions $E_{2}$ and $E_{3}$, corresponding to the second and the third sub-pulses of events with a multiplicity $M$ of at least 3 , shows that most triple-pulse sequences fit with the picture of consecutive $30 \mathrm{keV}$ and $138 \mathrm{keV}$ transitions (Figure 10). The figure visualizes the energy window used to select D3C events for further analyses. In addition, the energy deposition comprised in the primary pulse component was limited to $E_{1}<2 \mathrm{MeV}$. This cut had only little effect on the rate of identified D3C events but reduced the random background.

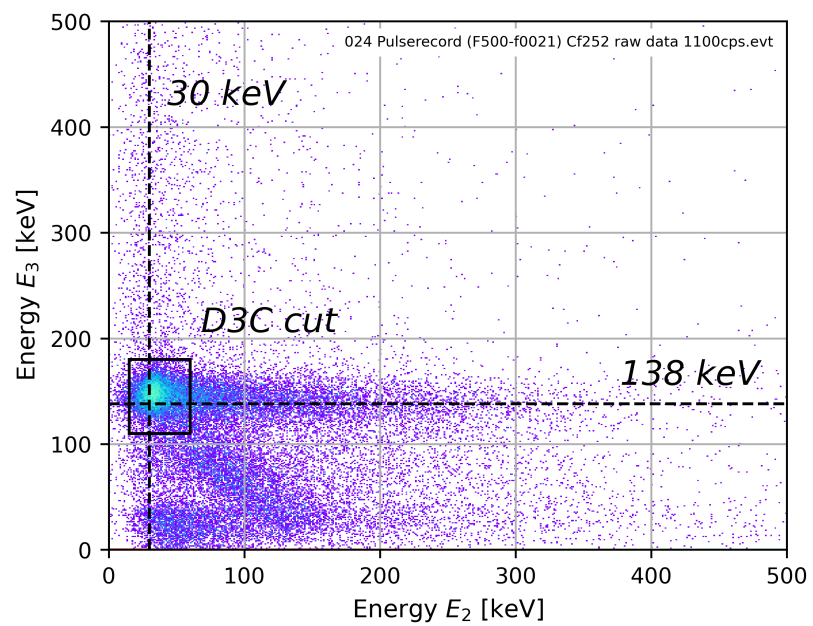

Figure 10. Energy depositions $E_{2}$ and $E_{3}$ assigned to the $2^{\text {nd }}$ and $3^{\text {rd }}$ signal components, respectively, plotted for all events of Run_024 with a multiplicity $M \geq 3$. The data were measured with the $\varnothing 2$ " $\times 2$ " $\mathrm{NaI}(\mathrm{Tl})$ scintillator, exposed to the ${ }^{252} \mathrm{Cf}$ neutron source, at a detector load of $1.1 \mathrm{kcps}$.

As a next step, the time delays $t_{21}=t_{2}-t_{1}, t_{32}=t_{3}-t_{2}$, and $t_{31}=t_{3}-t_{1}$ were determined for all $\mathrm{D} 3 \mathrm{C}$ events from the trigger points $t_{k}$ provided by the signal processing algorithm for the respective signal components. Delay time distributions obtained for the $\mathrm{NaI}(\mathrm{Tl})$ detector exposed to the neutron source are shown in Figure 11. The $t_{21}$ and the $t_{32}$ spectra were fitted by $f(t)=A \cdot e^{-\lambda t}+B$ representing an exponential decay plus a constant background. The half-lives of $175 \pm 1 \mathrm{~ns}$ and $867 \pm 18 \mathrm{~ns}$ derived from the fitted decay constants are in excellent agreement with the half-lives of the $167.4 \mathrm{keV}$ and the $137.8 \mathrm{keV}$ states. This confirms the interpretation of $\mathrm{D} 3 \mathrm{C}$ events as signals from de-excitation cascades in ${ }^{128}$ I involving both isomeric states.

Figure 12 compares the $t_{32}$ distribution of delayed triple coincidences (D3C events) with the $t_{21}$ distribution of delayed double coincidences (D2C events) extracted from the same measurement. Both delays result from the decay of the $137.8 \mathrm{keV}$ state. The slightly increased half-life from the D2C data fit may indicate the admixture of unresolved triple events: If a $30 \mathrm{keV}$ transition occurred, the corresponding delay adds to the delay of the subsequent $138 \mathrm{keV}$ signal. The $t_{31}$ distribution in Figure 11 illustrates this effect of delayed level feeding. If the $30 \mathrm{keV}$ component is missed by the pulse processing, either because of a pulse onset falling in the lead integration range (cf. Section III.B), or because of a signal vanishing in the tail of the primary signal (cf. Figure 4, Figure 7), the unresolved triple signal appears as D2C event with increased delay $t_{21}$ of the $138 \mathrm{keV}$ sub-pulse.

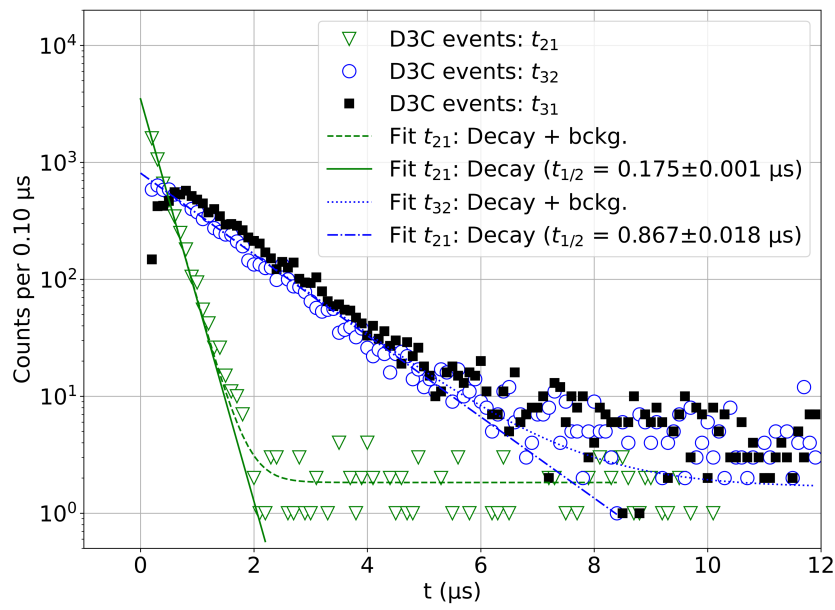

Figure 11. Delay time distributions of the delayed triple-coincidence events comprised in Run_024: $t_{21}, t_{31}$, and $t_{32}$ denote time delays between $2^{\text {nd }}$ and $1^{\text {st }}, 3^{\text {rd }}$ and $1^{\text {st }}$, and $3^{\text {rd }}$ and $2^{\text {nd }}$ signal components, respectively (cf. Figure 4 ). Fits with an exponential and constant background nicely reproduce the expected half-lives of isomeric levels in ${ }^{128} \mathrm{I}$.

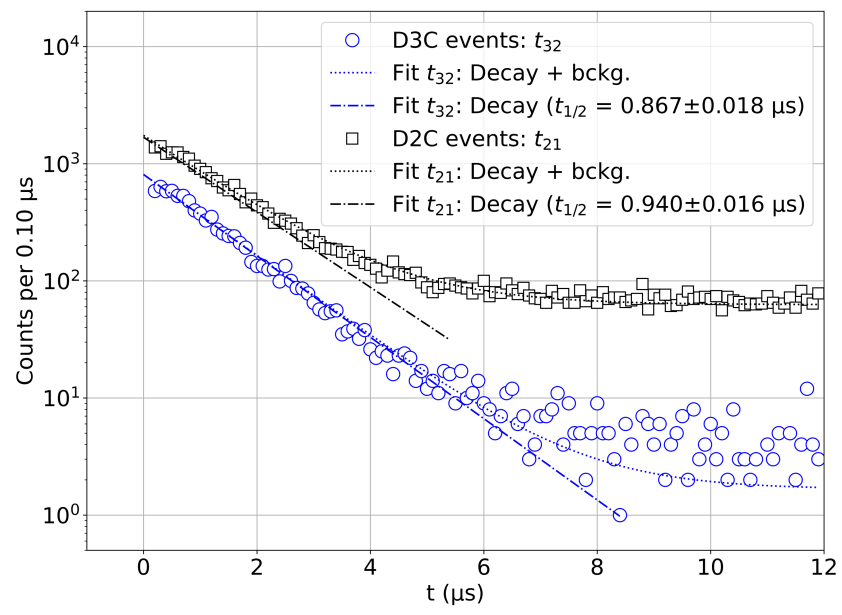

Figure 12. Delay time distributions assigned to the $138 \mathrm{keV}$ decay in the D2C and D3C events of Run_024. Fits with an exponential and constant background are compatible with the $845 \mathrm{~ns}$ half-life of the $137.8 \mathrm{keV}$ state in ${ }^{128} \mathrm{I}$.

The background in the delay time distributions is caused by random pulse pileups. The probability $d P$ that a pileup pulse appears after the primary pulse in an interval $d t$ at time $t$ is $d P=r \cdot e^{-r t} d t$, where $r$ denotes the mean detection rate [24]. It is evident that (1) the pileup probability and thus the random background increases with $r$, and (2) the assumption of a constant background is reasonable, as long as $r t \ll 1$. The range for background fitting comprises about $10 \mu \mathrm{s}$ (Figures 11-12), which translates to $r \ll 100 \mathrm{kcps}$. Fit functions with an exponential background reflecting the actual event rate are obviously more appropriate if the detector load reaches or exceeds the $10 \mathrm{kcps}$ level. 
For practical applications the number of neutron counts, i.e., the number of (true) delayed double- and triple-coincidences comprised in a dataset, must be quantified. This has been done in two different ways:

(i) Time cuts in $t_{21}$ or in $t_{21}$ and $t_{32}$ were applied for all detected $\mathrm{D} 2 \mathrm{C}$ or $\mathrm{D} 3 \mathrm{C}$ events, respectively. Events passing these filters, named D2T or D3T events, were counted to yield plain numbers $N_{D 2 T}$ and $N_{D 3 T}$, respectively.

(ii) The time distributions $t_{21}$ or $t_{32}$ corresponding to the $138 \mathrm{keV}$ transition of D2C or D3C events, respectively, were fitted with a function $f(t)=A \cdot e^{-\lambda_{138} t}+B$, where $\lambda_{138}=0.820 \mu \mathrm{s}^{-1}$ means the decay constant yielding a half-life of $0.845 \mu \mathrm{s}$. Effect and background counts were then derived by integrating the exponential and the background parts of the fit function in the time range 0 to $T$, which results in $N_{n m}=\frac{A}{\lambda_{138}}\left(1-e^{-\lambda T}\right)$ and $N_{b m}=B T$. The indices $m \in(2,3)$ denote D2C or D3C events, respectively.

Method (ii) provides separate measures for the true doubleor triple-event rates and for the random background, but it requires reasonable statistics in the time distributions to allow reliable fits. It is the method of choice for continuous neutron monitoring. If, however, few neutron counts must trigger an alarm at the shortest possible time scale, the plain counting method (i) is the only alternative. In this case it is important to find a tradeoff between effect count rate and background contribution. Having this in mind, the upper limits $T$ were chosen to yield $\lambda T=3$, which means $0.76 \mu \mathrm{s}$ for $t_{21}$ of D3C events $\left(\lambda=\lambda_{167}=3.96 \mu \mathrm{s}^{-1}\right)$, and $3.66 \mu$ s for $t_{21}$ of D2C and $t_{32}$ of $\mathrm{D} 3 \mathrm{C}$ events $\left(\lambda=\lambda_{138}\right)$. This value provides for reasonable effect-to-background ratios at the expense of a minor efficiency loss: The corresponding time interval contains about $95 \%$ of the decay contribution but reduces the background by $40 \%$ if compared with $\lambda T=5$ comprising $99 \%$ of the overall effect. For reasons of comparability, this upper limit was chosen for method (ii) as well. The lower limits for the time cuts applied in method (i) were set to $0.15 \mu \mathrm{s}$. This value is a bit smaller than the minimum detectible pulse delay $\Delta t_{\min }$ mentioned in Section III.B but rejects some events where fluctuations in the primary pulse tail cause immediate sub-pulse triggers.

\section{B. Trigger Threshold and Detection Efficiencies}

The probability to identify a delayed $30 \mathrm{keV}$ signal in the tail of a primary pulse, and thus the efficiency of detecting delayed triple coincidences, depends on the trigger threshold. Figures 8-9 already disclosed a "noise" bump at small delays $t_{21}$ and low energies $E_{2}$. Each wrong trigger caused by a statistical signal fluctuation disables the trigger search for $\Delta t_{\text {min }} \approx 160 \mathrm{~ns}$ (Section III.B) and prevents finding a true signal during this time. This reduces the $\mathrm{D} 3 \mathrm{C}$ detection efficiency at low trigger threshold settings. A higher threshold, however, might already cut off $30 \mathrm{keV}$ signals.

This effect was studied by analyzing simulated sets of

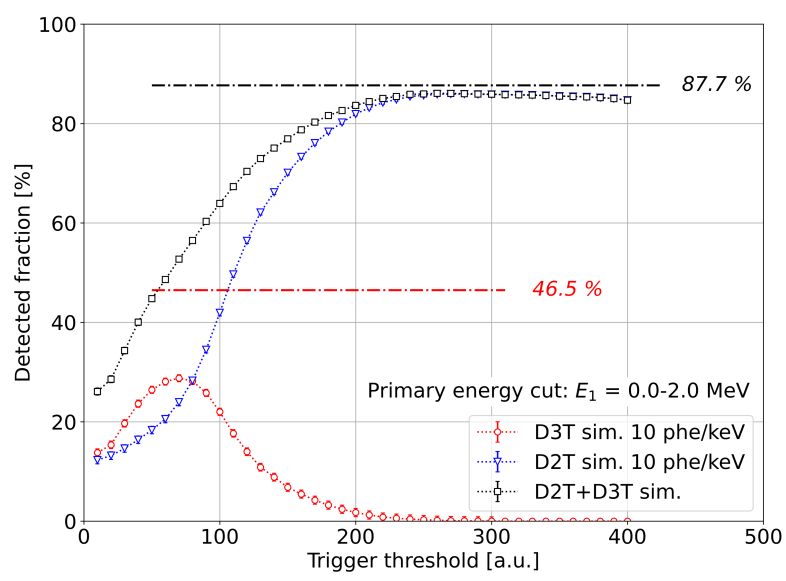

Figure 13. Fraction of delayed triple-coincidence signals, modeled with a photoelectron yield of $10 \mathrm{phe} / \mathrm{keV}$, which are identified as D2C or D3C counts, in dependence of the trigger threshold setting. Chain-dotted lines mark the upper limits given by the minimum detectible pulse delays and the decay time of the delayed transitions. The fraction of triple events misidentified as $\mathrm{D} 2 \mathrm{C}$ rises with the trigger threshold.

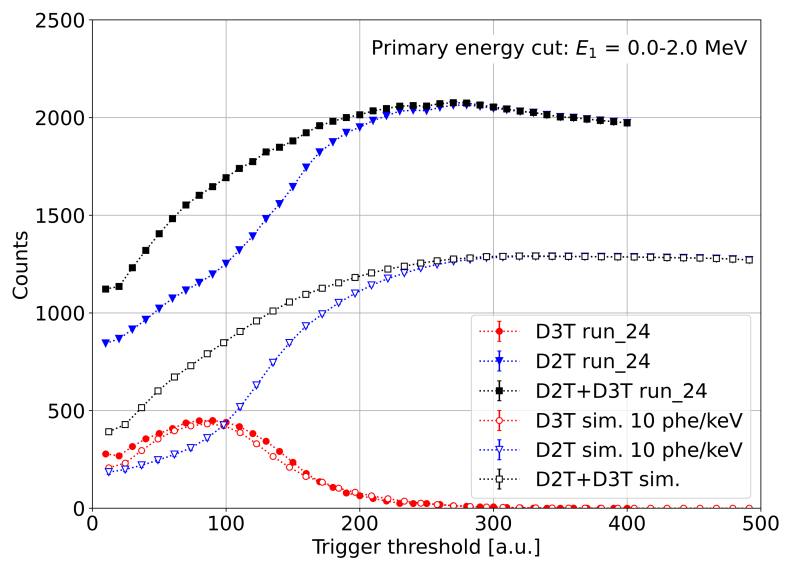

Figure 14. Delayed double (D2T) and triple (D3T) coincidence counts extracted from data measured with the $\mathrm{NaI}(\mathrm{Tl})$ detector at the neutron source. The dependencies on the trigger threshold are compared with efficiency curves for simulated triple pulses, scaled to fitting D3T curves (cf. text).

delayed triple-coincidence signals (Section III.E). Figure 13 shows an exemplary analysis of 25,600 triple signals modeled with a photoelectron yield of $10 \mathrm{phe} / \mathrm{keV}$. The line graphs represent fractions of the processed signals that were identified as D3T and D2T events, respectively, and the sum of both fractions, as a function of the trigger threshold setting. In accordance with the analysis of experimental data, only events with a primary energy deposition below $2 \mathrm{MeV}$ were considered. The fraction of identified D3T events reaches about $30 \%$ at optimum trigger threshold. Obviously, the $30 \mathrm{keV}$ sub-pulse is not found in a considerable fraction of all triple signals. Those are identified as D2C events if the subsequent $138 \mathrm{keV}$ sub-pulse is correctly identified. At higher trigger threshold, the D2T fraction almost reaches the theoretical limit given by the minimum detectible sub-pulse delay (chain-dotted line). The maximum possible D3T fraction is missed even at optimum trigger threshold. The sum of both D2T and D3T fractions suggests a noticeable rise of the stripping algorithm's dead time for trigger levels below 150, which is however far above the optimum for D3T detection. 
Figure 14 presents a similar analysis of signals measured with the $\mathrm{NaI}(\mathrm{Tl})$ detector at the neutron source. The signal processing was executed for different trigger level settings, and the numbers $N_{D 2 T}$ and $N_{D 3 T}$ of detected D2T and D3T events as well as their sum was plotted versus the threshold. The modeled curves of Figure 13 were adapted to this plot by (1) scaling the trigger threshold axis in accordance with the (slightly different) energy calibration factors of simulation and measurement, and (2) scaling the height of the simulated D3T efficiency curve to the measured $N_{D 3 T}$ values. The congruence of simulated and measured D3T curves is striking. In simulations, the corresponding shape depends a bit on the photoelectron yield assumed, but the best agreement is in fact achieved with $10 \mathrm{phe} / \mathrm{keV}$, which is a reasonable value for PMT with standard bialkali photocathode. This provides confidence in the corresponding prediction of D2T counts representing misinterpreted triple-coincidence signals, obtained by scaling the D2T fraction of Figure 13 with the same factors as the D3T curve. A comparison with the measured values suggests that about 1,300 of the 2,000 to 2,100 D2T events registered at optimum trigger settings for D2T detection are due to delayed triple coincidences. This means that about 2 of 3 gamma-ray cascades involving the $137.8 \mathrm{keV}$ (845 ns) state also comprise the $30 \mathrm{keV}$ transition feeding this level from the isomeric $167.4 \mathrm{keV}$ (175 ns) state.

\section{Measured Neutron Detection Yields}

Measurements performed with the $\varnothing 2$ " $\times 2$ " NaIL TM detector and with the common $\mathrm{NaI}(\mathrm{Tl})$ detector of the same size, each one exposed to gamma rays and neutrons or to gamma rays only, respectively, were analyzed to evaluate the neutron detection performance achievable with the different techniques. The trigger threshold for signal processing was set to 120 . This is not far from the optimum for delayed triplecoincidence counting but ensures an increased D2T detection efficiency at an acceptable loss of D3T events (cf. Figure 14). Numbers of delayed double or triple coincidences and random background contributions were determined as described in Section A above. In addition, ${ }^{6} \mathrm{Li}$ neutron-capture events and high-energetic neutron capture signals were counted as exemplified in Sections III.D-III.C. All numbers $N_{x}$ were then translated to count rates $r_{x}=r_{e v t} \cdot \frac{N_{x}}{N_{e v t}}$ by relating them to the actual detector load $r_{e v t}$, measured under identical conditions in normal RID mode (Section III.A), and to the overall number of events $N_{\text {evt }}$ analyzed offline. This approach bypasses the problem of determining precise system dead and live times for data acquisition in raw-data mode.

The results are compiled in Table I. Standard deviations stated there were derived from variances of the fit parameters. The table confirms the intuitive presumption that delayed triple-coincidence counting provides a lower rate of neutron detections but a better suppression of random background than delayed double-coincidence counting. For the latter, the background fraction rises from about $10 \%$ at $1.1 \mathrm{kcps}$ detector load (Run_24, Run_25) to more than 50\% at the (still moderate) load of $3.3 \mathrm{kcps}$ (Run_21, Run_22). On the other hand, the sum of D2T and D3T count rates achieved with the $\mathrm{NaI}(\mathrm{Tl})$ detector are of the same order of magnitude (though by far not as high) as the ${ }^{6} \mathrm{Li}$ capture rate measured with $\mathrm{NaIL}^{\mathrm{TM}}$. If the rate $r_{N C D}$ of high-energy capture-gamma events is included, the overall, background-corrected detection rate of ${ }^{127}$ I neutron captures reaches about 17 cps (Run_024), which is almost half of the ${ }^{6} \mathrm{Li}$ capture rate measured in Run_021.

\section{Load Dependence of Random Background Contributions}

The higher the detector load, the higher is the probability of random pulse pileups. Those contaminate rates and time distributions of delayed double- and triple-coincidence events. A set of measurements, taken at constant neutron flux but rising gamma load generated by supplementary ${ }^{137} \mathrm{Cs}$ and ${ }^{232} \mathrm{Th}$ sources, was analyzed to quantify this effect. The analysis was performed in the same way as in Section C.

Figure 15 shows the load dependence of the D2T and D3T count rates $r_{D 2 T}$ and $r_{D 3 T}$ together with effect $\left(r_{n 2}\right.$ and $\left.r_{n 3}\right)$ and the combined effect plus background rates $\left(r_{n 2}+r_{b 2}\right.$ and $\left.r_{n 3}+r_{b 3}\right)$ derived from fits of the delay time distributions comprising the background and an exponential related with the $138 \mathrm{keV}$ ( $845 \mathrm{~ns}$ ) transition. The D2T count rate rises immediately at detector loads above $1 \mathrm{kcps}$ and multiplies tenfold around $10 \mathrm{kcps}$. This behavior is well reproduced by the sum of effect and background rates $r_{n 2}+r_{b 2}$. The corresponding effect rate $r_{n 2}$ indicates that fitting of the delay

TABLE I

COMPARISON OF NEUTRON COUNT RATES AND RANDOM BACKGROUND OBTAINED WITH A NaiL TM AND A COMMON NaI(Tl) DETECTOR

\begin{tabular}{|c|c|c|c|c|c|c|c|}
\hline \multicolumn{2}{|c|}{ Quantity } & Acronym & Unit & \multicolumn{2}{|c|}{$\emptyset 2 " \times 2 " \mathrm{NaIL}^{\mathrm{TM}}$} & \multicolumn{2}{|c|}{$\emptyset 2 ” \times 2 ” \mathrm{NaI}(\mathrm{Tl})$} \\
\hline \multicolumn{4}{|c|}{ Measurement } & Run_21 & Run_22 & Run_24 & Run_25 \\
\hline \multicolumn{4}{|l|}{ Sources } & ${ }^{252} \mathrm{Cf},{ }^{232} \mathrm{Th}$ & ${ }^{137} \mathrm{Cs},{ }^{232} \mathrm{Th}$ & ${ }^{252} \mathrm{Cf}$ & ${ }^{232} \mathrm{Th}$ \\
\hline \multicolumn{2}{|l|}{ Events } & $N_{e v t}$ & $10^{6}$ & 3.840 & 0.256 & 3.840 & 3.840 \\
\hline \multicolumn{2}{|c|}{ NCD count rate } & $r_{N C D}$ & $\mathrm{cps}$ & 7.48 & 0.28 & 9.54 & 0.18 \\
\hline \multicolumn{2}{|c|}{$\mathrm{D} 2 \mathrm{~T}$ count rate } & $r_{D 2 T}$ & $\mathrm{cps}$ & 9.87 & 5.79 & 6.27 & 0.62 \\
\hline $\mathrm{D} 2 \mathrm{C}$ fit & Effect & $r_{n 2}$ & $\mathrm{cps}$ & $4.96 \pm 0.10$ & $1.01 \pm 0.25$ & $5.90 \pm 0.09$ & $0.01 \pm 0.01$ \\
\hline \multirow{3}{*}{ D3C fit } & Effect + bckg. & $r_{n 2}+r_{b 2}$ & cps & $10.35 \pm 0.52$ & $6.04 \pm 1.51$ & $6.63 \pm 0.12$ & $0.66 \pm 0.06$ \\
\hline & Effect & & cps & $1.34 \pm 0.03$ & $-0.03 \pm 0.03$ & $1.65 \pm 0.02$ & $0.00 \pm 0.00$ \\
\hline & Effect + bckg & $r_{n 3}+r_{b 3}$ & cps & $1.43 \pm 0.05$ & $0.08 \pm 0.20$ & $1.67 \pm 0.03$ & $0.01 \pm 0.01$ \\
\hline
\end{tabular}




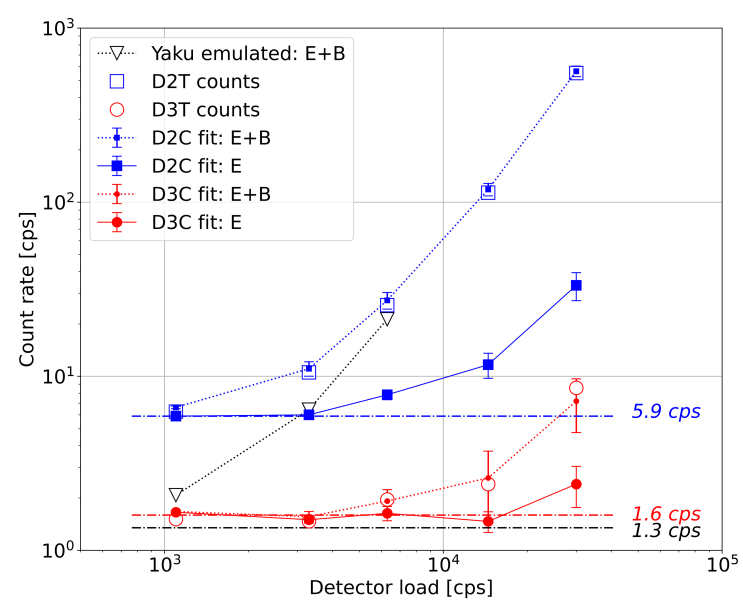

Figure 15. Dependence of measured neutron count rates on the detector load. Direct D2T and D3T count rates agree well with the sum of effect (E) and background (B) rates derived from fits of the delay-time distributions. Yaku denotes the rate emulated for the original delayed-coincidence technique as proposed in [16]. Chain-dotted lines mark the most reliable backgroundcorrected effect rates derived from runs with low background contributions.

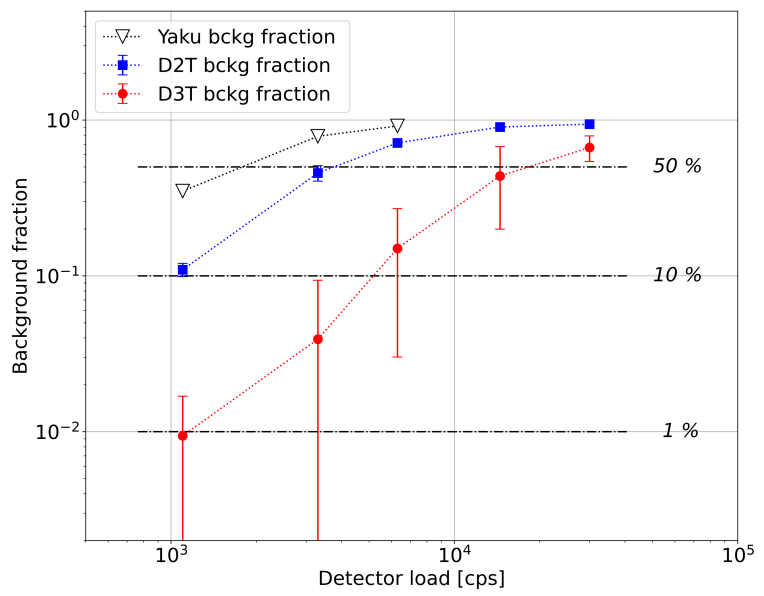

Figure 16. Background fraction in D2T and D3T count rates as derived from fits of the delay-time distributions. Yaku denotes data emulated for the original delayed-coincidence technique as proposed in [16].

time distribution eliminates random pileups quite well for loads up to 3-4 kcps.

The D3T count rate starts deviating from the expected constant level above $5 \mathrm{kcps}$. Here, the fit method provides reliable effect rates up to at least $15 \mathrm{kcps}$. Even at $30 \mathrm{kcps}$ the difference to the expected value (chain-dotted line) is within two standard deviations. This confirms that the delayed triplecoincidence method is more robust against disturbing gamma background than the delayed double-coincidence technique.

Figure 16 provides another view by showing the random background fractions comprised in the D2T and D3T count rates as a function of the detector load. The chain-dotted lines mark background levels of $1 \%, 10 \%$, and $50 \%$, respectively. The latter can be seen as reasonable upper limit and is reached around 3-4 kcps or 15-20 kcps for the delayed double- and triple-coincidence techniques, respectively.

Both figures include a comparison with the effect and background rates achievable with the method introduced by Yakushev et al. [16]. The corresponding effect counts were emulated by integrating the fitted exponentials $A \cdot e^{-\lambda_{138} t}$ of the individual runs from $t_{0}=1.8 \mu \mathrm{s}$ (the trigger hold-off specified in [16]) to $t_{1}=t_{0}+T$ instead of 0 to $T$ while keeping $\lambda T=3$ for the length of the integration interval (cf. Section A). The obtained effect rate of $1.3 \mathrm{cps}$ is lower than that of the true triple-coincidence events $(1.6 \mathrm{cps})$ detected with the D3T counting technique, while the background rate is the same as for D2T counting. This results in an unsatisfactory background fraction already at detector loads as low as $1 \mathrm{kcps}$ (Figure 16). An effective reconstruction of piled-up signal components is obviously mandatory for using delayed coincidences to measure neutrons in real applications.

\section{E. Energy spectra of Primary Signal Components}

Energy spectra related with the prompt signal components of delayed double- or triple-coincidence events are presented and compared with the corresponding single-event spectrum in Figure 17. The graph reveals the upper energy limit of $2 \mathrm{MeV}$ introduced for the $\mathrm{D} 2 \mathrm{C}$ and $\mathrm{D} 3 \mathrm{C}$ event analysis (Section A) and evidences the minor efficiency loss associated with this cut. It also discloses a clear line structure in the $E_{1}$ spectra of the delayed-coincidence events below $500 \mathrm{keV}$.

Figure 18 shows an expanded view of this range. The gamma lines marked in the spectrum of D2T events are in reasonable correspondence with the strongest feeding transitions of the $137.8 \mathrm{keV}$ state as given by Sakharov et al. [17] (markers in the figure), if the light excess caused by the nonproportionality of $\mathrm{NaI}(\mathrm{Tl})$ scintillators is taken into account. The peak at $30 \mathrm{keV}$ can be explained by neutroncapture events where the prompt part of the cascade is missed by the detector, while a delayed $30 \mathrm{keV}$ transition feeding the $137.8 \mathrm{keV}$ level generates the first sub-pulse.

The 167.4 state is, according to [17], basically fed from $376 \mathrm{keV}\left(4^{-}\right)$by subsequent $142 \mathrm{keV}$ and (partially converted) $67 \mathrm{keV}$ transitions with a sum energy of $209 \mathrm{keV}$. As one of the gamma rays may pass the detector without interaction, this feeding chain provides an explanation for the three dominant peaks in the spectrum associated with D3T events. These transitions might also contribute to the peak structure formed by $\mathrm{D} 2 \mathrm{~T}$ events: If the $30 \mathrm{keV}$ transition is missed by the pulse processing algorithm, its energy contributes to the integral representing the prompt pulse component. Corresponding counts would contribute to the D2T spectrum but would be shifted by $30 \mathrm{keV}$. By chance, the resulting energies are close to that of transition promptly feeding the $137.8 \mathrm{keV}$ level.

Independent of a physical interpretation, the distinct, almost isolated lines related with delayed-coincidence events provide means for further background reduction. Energy windows in $E_{1}$ comprising these peaks could be applied to reject events with primary energies rarely appearing in D2T or D3T events. This would add further and quite sharp selection criteria for an unambiguous identification of gamma-ray cascades following neutron capture in ${ }^{127} \mathrm{I}$.

\section{DISCUSSION}

The results highlight the potential of $\mathrm{NaI}(\mathrm{Tl})$ detectors for detecting thermal neutrons even in the presence of ambient 


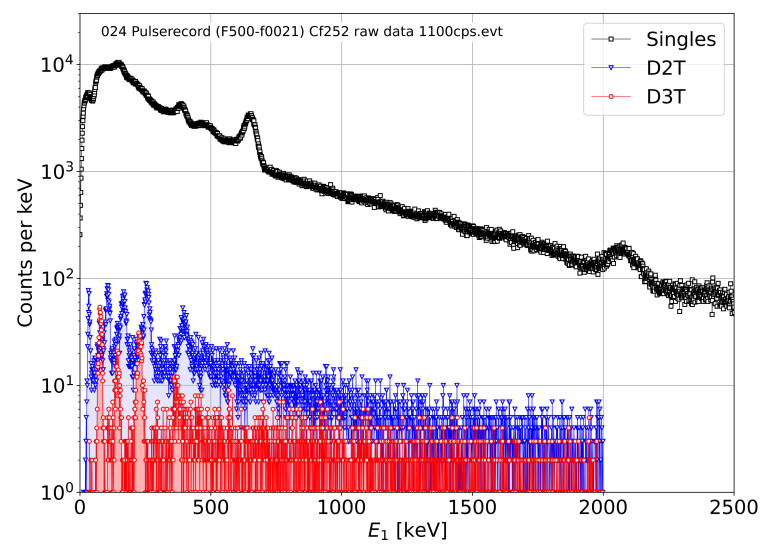

Figure 17. Energy deposition related with the primary signal component of single, D2T, and D3T events comprised in Run_24. The low-energy part of spectra representing delayed-coincidence events exhibit a clear line structure.

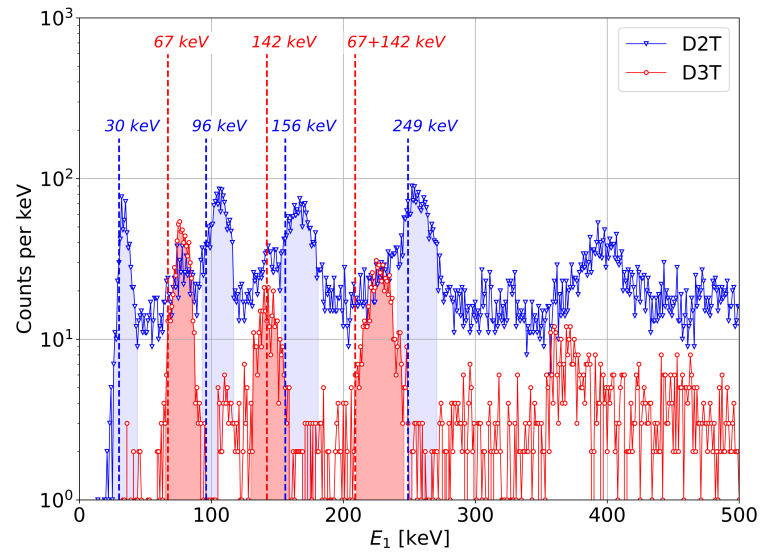

Figure 18. Energy deposition related with the primary signal component of D2T and D3T events comprised in Run_24. The line structures are in reasonable correspondence with known feeding transitions of the isomeric states, if the light excess caused by the nonproportionality of $\mathrm{NaI}(\mathrm{Tl})$ scintillators in the low-energy range is taken into account (cf. text).

gamma radiation. Though the performance of excellent (but expensive) neutron-gamma scintillators as NaIL ${ }^{\mathrm{TM}}$ is not in reach, radiation detection systems based on $\mathrm{NaI}(\mathrm{Tl})$ scintillators could be provided with remarkable neutron sensitivity by just adding an appropriate digital signal analysis.

In potential applications there are two major scenarios distinguished by the measuring time and temporal pattern:

(i) Neutron monitoring in facilities or in the environment often means logging a time-averaged measure of the respective neutron flux in regular periods. If an immediate response to flux variations at low levels is not required, there is enough time to collect some statistics. This scenario could be perfectly served with the delayedcoincidence techniques and delay-time spectrum fitting. The effects discussed above provide comprehensive tools to adapt the technique to the expected levels of neutron flux and gamma-ray background, and to the period of data logging. The choice of double- or triplecoincidence events including a corresponding adaptation of the trigger threshold, the selection of time cuts, and the possible application of additional energy cuts to the prompt signal component open a wide parameter space to find the best tradeoff between neutron detection efficiency and background rejection.

(ii) Instruments for security applications with (optional) neutron detection capabilities are required to generate neutron alarms within few seconds in response to dynamic changes of low-intensity neutron fields. To be compliant with the corresponding standards [1] [2], the alarming must be based on a few single neutron counts even if efficient, state-of-the-art neutron detectors are used. This constraint requires plain counting instead of spectrum fitting if delayed-coincidence techniques are to be applied. The numbers in Table I demonstrate the advantage of NaiL ${ }^{\text {TM }}$ detectors in this case. Nevertheless the neutron sensitivity achievable with common $\mathrm{NaI}(\mathrm{Tl})$ differs only gradually but not by an order of magnitude, if the available techniques - NCD principle, delayed double- and triple-coincidence methods - are combined.

To our opinion, $\mathrm{NaI}(\mathrm{Tl})$-based instruments with limited neutron detection capabilities are useful even if they do not fully comply with the neutron-alarming requirements for RIDs or BRDs with optional neutron detector. The drawback concerns basically the time needed to trigger a neutron alarm. However, statistics and thus the information about effect and background contributions improve with the measuring time. In case of a gamma alarm, the neutron sensitivity could be used to verify or deny the presence of neutrons in parallel to the gamma spectrum acquisition, which would add more confidence for the classification of suspect sources.

The event rates measured with the NaIL ${ }^{\mathrm{TM}}$ detector allow estimating the fraction of neutron-capture gamma cascades involving either one or both long-lived states in ${ }^{128}$ I. Table I states D2C and D3C effect rates (fit results) of 4.96 and $1.34 \mathrm{cps}$, respectively. About $2 / 3$ of all counted D2C events are due to triple events with a missed $30 \mathrm{keV}$ sub-pulse (Section IV.B), which leaves a true D2C rate of $1.65 \mathrm{cps}$. Simulations predict trigger efficiencies of $60 \%$ and $25 \%$ for true double and triple events at a trigger threshold scaled to that used for the data analysis (Figures 13-14). This yields true double and triple rates of 2.76 and 5.36, summing to $8.12 \mathrm{cps}$. The measured ${ }^{6} \mathrm{Li}$ capture rate was $38.7 \mathrm{cps}$ in this measurement (Table I). It is equivalent with the true ${ }^{6} \mathrm{Li}$ capture rate as the corresponding detection efficiency is close to $100 \%$. The true neutron-capture rate in ${ }^{127} \mathrm{I}$ can be estimated by multiplying this number with the ratio of the neutron-capture cross sections, scaled with the relative occurrence of the nuclides in the crystal. If we suppose a ${ }^{6} \mathrm{Li}$ concentration of $1 \mathrm{at} \%$ in the NaIL ${ }^{\mathrm{TM}}$ crystal as suggested by Yang et al. [10], we obtain $38.7 \mathrm{cps} \times 6.2 \mathrm{~b} /(0.01 \times 940 \mathrm{~b})$ $=25.5 \mathrm{cps}$. This means about one third $(8.12 / 25.5)$ of all neutron capture events in ${ }^{127}$ I generate gamma-ray cascades involving the isomeric $137.8 \mathrm{keV}$ ( $845 \mathrm{~ns}$ ) state, and about two thirds of those (representing about $20 \%$ of all capture events) involve the $167.4 \mathrm{keV}$ (175 ns) state as well.

Despite some impressive results, the pulse processing used here cannot fully deploy this potential. The $160 \mathrm{~ns}$ dead time 
after trigger (Section III.B) means a tremendous improvement to the $1.8 \mu$ s trigger holdoff in Yakushev's paper [16] but still cuts off most delayed triple-coincidence events. The low trigger threshold, indispensable for finding a $30 \mathrm{keV}$ signal in the tail of the primary pulse, entails random triggers, increases the dead time, and reduces the detection efficiency for all delayed-coincidence events. An optimized pulse processing with separate digital filters feeding "fast" and "slow" trigger channels might leave room for improvements. For the given algorithm, the intuitive assumption that an increased photoelectron yield would noticeably improve the detection efficiencies could not be confirmed by simulations.

It is worth noting that delayed pulse components affect common pulse shape parameters as used for charged-particle identification and discrimination, e.g., in NaIL ${ }^{\mathrm{TM}}$ detectors. The faint ridge below the bulk of gamma-ray events in Figure 5 is due to signals where the delayed signal component(s) add a constant charge to the pulse tail, which shifts them away from the main gamma crest in the PSD plot. Corresponding observations have been made by other authors (e.g. in [25]), but have not been interpreted correctly.

\section{CONCLUSION}

Searching digitized detector signals for piled-up delayed components with distinct energy and delay time signatures is a smart method to provide common $\mathrm{NaI}(\mathrm{Tl})$ detectors with additional neutron detection capabilities at no extra cost. This technique nicely complements the idea of the Neutron Capture Detector (NCD) analyzing events with energy depositions above the range of common gamma-ray energies. In combination, both approaches can provide half of the neutron sensitivity offered by a commercial ${ }^{6} \mathrm{Li}$ co-doped $\mathrm{NaI}(\mathrm{Tl})$ (NaIL ${ }^{\mathrm{TM}}$ ) scintillator of the same size, at the price of higher and load-dependent background contributions. Delayedcoincidence techniques are most suitable for neutron monitoring or long-term measurements, where the statistics of the acquired delay-time distributions allows separate fitting of the effect and background contributions. In this case, the thermal neutron flux can be quantified in parallel to gammaray spectroscopy at overall detector loads exceeding $10 \mathrm{kcps}$.

\section{REFERENCES}

[1] American National Standard Performance Criteria for Handheld Instruments for the Detection and Identification of Radionuclides. IEEE/ANSI N42.34-2021, American National Standards Institute, 2021. https://standards.ieee.org/standard/N42 34-2021.html

[2] American National Standard Performance Criteria for Backpack-Based Radiation-Detection Systems Used for Homeland Security. IEEE/ANSI N42.53-2021, American National Standards Institute, 2021. https://standards.ieee.org/standard/N42 53-2021.html

[3] R. T. Kouzes, "The ${ }^{3}$ He Supply Problem," Report PNNL-18388 (2009). https://www.pnnl.gov/main/publications/external/technical_reports/PNN L-18388.pdf

[4] A. J. Hurd and R. T. Kouzes, "Why new neutron detector materials must replace helium-3,” Eur. Phys. J. Plus (2014) 129: 236. http://dx.doi.org/10.1140/epjp/i2014-14236-6

[5] R. T. Kouzes, A. T. Lintereur, and E. R. Siciliano: "Progress in alternative neutron detection to address the helium-3 shortage," Nucl. Instrum. Methods Phys. Res. A 784 (2015) 172-175. https://doi.org/10.1016/j.nima.2014.10.046
[6] G. F. Knoll, "Radiation detection and Measurement", $4^{\text {th }}$ edition, John Wiley \& Sons, Inc., 2010, pp. 521-523.

[7] A. Bessiere, P. Dorenbos, C. W. E. van Eijk, K. W. Krämer, and H. U. Güdel, "New Thermal Neutron Scintillators: $\mathrm{Cs}_{2} \mathrm{LiYCl}_{6}: \mathrm{Ce}^{3+}$ and $\mathrm{Cs}_{2} \mathrm{LiYBr}_{6}: \mathrm{Ce}^{3+}$," IEEE Trans. Nucl. Sci. 51 (5) (2004) 2970-2972. https://doi.org/10.1109/TNS.2004.834957

[8] J. Glodo, W. M. Higgins, E. V. D. van Loef, and K. S. Shah, "Scintillation Properties of 1 Inch $\mathrm{Cs}_{2} \mathrm{LiYCl}_{6}: \mathrm{Ce}$ Crystals," IEEE Trans. Nucl. Sci. 55 (3) (2008) 1206-1209. https://doi.org/10.1109/TNS.2007.913467

[9] U. Shirwadkar, J. Glodo, E. V. van Loef, R. Hawrami, S. Mukhopadhyay, A. Churilov, W. M. Higgins, and K. S. Shah, "Scintillation properties of $\mathrm{Cs}_{2} \mathrm{LiLaBr}_{6}$ (CLLB) crystals with varying $\mathrm{Ce}^{3+}$ concentration," Nucl. Instrum. Methods Phys. Res. A 652 (2011) 268-270. https://doi.org/10.1016/j.nima.2010.08.050

[10] K. Yang, P. R. Menge, and V. Ouspenski, "Li Co-Doped NaI:Tl (NaIL) - A Large Volume Neutron-Gamma Scintillator with Exceptional Pulse Shape Discrimination," IEEE Trans. Nucl. Sci. 64 (8) (2017) 2406-2413. https://doi.org/10.1109/TNS.2017.2721398

[11] Database of Prompt Gamma Rays from Slow Neutron Capture for Elemental Analysis; IAEA, Vienna, 2007, ISBN 92-0-101306-X. https://www.iaea.org/publications/7030/database-of-prompt-gammarays-from-slow-neutron-capture-for-elemental-analysis

[12] M. A. Islam, T. J. Kennett, and W. V. Prestwich, "A Study of the ${ }^{127} \mathrm{I}(n, \gamma)^{128} \mathrm{I}$ Reaction,” Z. Phys. A Atomic Nuclei 335, 173-182 (1990). https://doi.org/10.1007/BF01294472

[13] G. Pausch, C. M. Herbach, and J. Stein, "Apparatus and Method for Neutron Detection with Neutron-Absorbing Calorimetric Gamma Detectors," U.S. Patent Application 20120074326 (2009). https://patents.justia.com/patent/20120074326\#history

[14] G. Pausch, C. M. Herbach, D. Mitchell, R. Lentering, and J. Stein, "Neutron detection based on capture-gamma sensing and calorimetry," Proc. SPIE 8382, Active and Passive Signatures III, 838209 (2012). https://doi.org/10.1117/12.924082

[15] G. Pausch, C. M. Herbach, Y. Kong, R. Lentering, C. Plettner, K. Roemer, F. Scherwinski, J. Stein, P. Schotanus, and T. Wilpert, "Neutron detection by measuring capture gammas in a calorimetric approach," Nucl. Instrum. Methods Phys. Res. A 652 (2011) 374-380. http://dx.doi.org/10.1016/j.nima.2010.08.103

[16] E. Yakushev, S. Rozov, A. Drokhlyansky, D. Filosofov, Z. Kalaninova, V. Timkin, and D. Ponomarev, "Sensitive neutron detection method using delayed coincidence transitions in existing iodine-containing detectors,” Nucl. Instrum. Methods Phys. Res. A 848 (2017) 162-165. http://dx.doi.org/10.1016/j.nima.2016.12.022

[17] S. L. Sakharov, V. L. Alexeev, I. A. Kondurov et al., "Low-lying ${ }^{128} \mathrm{I}$ excited states from the $(\mathrm{n}, \gamma)$ reaction," Nucl. Phys. A 528 (1991) 317347. https://doi.org/10.1016/0375-9474(91)90092-K

[18] Z. Elekes and J. Timar, "Nuclear Data Sheets for A $=128$," Nuclear Data Sheets 129 (2015) 191-436. http://dx.doi.org/10.1016/j.nds.2015.09.002

[19] G. F. Knoll, "Radiation detection and Measurement", $4^{\text {th }}$ edition, John Wiley \& Sons, Inc., 2010, p. 238.

[20] M. Moszynski, J. Zalipska, M. Balcerzyk, M. Kapusta, W. Mengesha, and J. D. Valentine, "Intrinsic energy resolution of $\mathrm{NaI}(\mathrm{Tl})$," Nucl. Instrum. Methods Phys. Res. A 484 (2002) 259-269. https://doi.org/10.1016/S0168-9002(01)01964-7

[21] J. Stein, A. Kreuels, Y. Kong, R. Lentering, K. Ruhnau, F. Scherwinski, and A. Wolf, "Experiment and modeling of scintillation photon-counting and current measurement for PMT gain stabilization," Nucl. Instrum. Methods Phys. Res. A 782 (2015) 20-27. https://doi.org/10.1016/i.nima.2015.01.101

[22] J. B. Birks, "The Theory and Practice of Scintillation Counting," Pergamon Press, Oxford, Germany: Vieweg \& Sohn GmbH, Braunschweig, First edition 1964, Reprinted 1967, pp. 148-151.

[23] M. Moszyński, A. Syntfeld-Każuch, L. Swiderski, M. Grodzicka, J. Iwanowska, P. Sibczyński, and T. Szczęśniak, "Energy resolution of scintillation detectors," Nucl. Instrum. Methods Phys. Res. A 805 (2016) 25-35. https://doi.org/10.1016/j.nima.2015.07.059

[24] G. F. Knoll, "Radiation detection and Measurement", $4^{\text {th }}$ edition, John Wiley \& Sons, Inc., 2010, p. 99-100.

[25] L. Caifeng, Q. Jianguo, X. Jun, Z. Tonghua, L. Xinxin, A. Li, M. Yunfeng, Z. Pu, S. Junjie, J. Li, W. Mei, and H. Zijie, "Particle discrimination and fast neutron response for a NaIL:Tl and NaI:Tl scintillator detector," Nucl. Instrum. Methods Phys. Res. A 978 (2020) 164372. https://doi.org/10.1016/j.nima.2020.164372 